\title{
Centipedes (Chilopoda) from the Rostov-on-Don Region, southern Russia
}

\section{Губоногие многоножкки (Chilopoda) из Ростовской области (юг России)}

\author{
R.V. Zuev ${ }^{1}$, A.P. Evsyukov ${ }^{2}$ \\ P.В. Зуев ${ }^{1}$, А.П. Евсюков ${ }^{2}$
}

${ }^{1}$ Северокавказский федеральный университет, ул. Пушкина 1, г. Ставрополь 355009, Россия.
${ }^{1}$ North Caucasian Federal University, Pushkina Str. 1, Stavropol 355009, Russia.
${ }^{2}$ «Классический лицей № 1», ул. Балакирева 32, Ростов-на-Дону 344004, Россия.
${ }^{2}$ “Classical lyceum No. 1”, Balakireva Street 32, Rostov-on-Don 344004, Russia.

KEY WORDS: Chilopoda, faunistics, Rostov-on-Don Region, southern Russia.

КЛЮЧЕВЫЕ СЛОВА: Chilopoda, фаунистика, Ростовская область, юг России.

ABSTRACT. The centipedes fauna of the Rostovon-Don Region is shown to comprise at least 27 species, six of which are new to the region, including one new to the fauna of Russia: Strigamia cf. transsilvanica (Verhoeff, 1928). Faunistic records of all of the species are presented and mapped in the region.

РЕЗЮМЕ. Фауна губоногих многоножек Ростовской области включает, по крайней мере, 27 видов, из них шесть впервые приведены для исследуемой территории, в том числе новый для фауны России Strigamia cf. transsilvanica (Verhoeff, 1928). Представлены фаунистические находки и карты с указанием распространения этих видов в регионе.

\section{Introduction}

The steppe zone of Russia, including the Rostov-onDon Region, is long known to have been subjected to considerable anthropogenic transformations. These transformations are reflected throughout soil fauna, including centipedes.

The earliest records of Chilopoda from the Rostovon-Don Region are contained in a number papers by Folkmanova and co-authors [Folkmanova, 1956, 1958; Ghilarov, Folkmanova, 1957; Folkmanova, Dobroruka, 1960], where as many as 12 species were reported: Escaryus retusidens Attems, 1904, E. ornatus Folkmanova, 1956 (referred to as E. retusidens ornatus), Diphyonyx sukacevi (Folkmanova, 1956) (referred to as Brachygeophilus sukacevi), Lithobius crassipes L. Koch, 1862, L. curtipes C.L. Koch, 1847 (referred to as Monotarsobius crassipes and M. curtipes, respectively), Cryptops anomalans Newport, 1844, Henia bicarinata (Meinert, 1870), Clinopodes escherichii (Verhoeff, 1896), Pachymerium ferrugineum (C.L. Koch, 1835), Geophilus flavus (De Geer, 1778) (referred to as two species: Schizotaenia ornata Folkmanova, Dobroruka, 1960 and S. tristanica (Attems, 1928)), Hessebius multicalcaratus Folkmanova, 1958 and an undescribed lithobiid species, "Lithobius quasipachypus Folk.", nomen nudum.
The most complete information about the centipedes of the region is presented in the papers of Minoranskiy [1975, 1977]. He cited 17 species, including six newly recorded: Scutigera coleoptrata (Linnaeus, 1758), Lamyctes emarginatus (Newport, 1844) (referred to as $L$. fulvicornis Meinert, 1868), Lithobius forficatus (Linnaeus, 1758), L. sseliwanoffi (Garbowski, 1897) (referred to as M. sseliwanoffi), Scolopendra cingulata Latreille, 1789 and Diphyonyx garutti (Folkmanova et Dobroruka, 1960) (referred to as Brachygeophilus sukacevi garutti).

Three further species were recorded about the same time: Clinopodes flavidus C.L. Koch, 1847 (referred to as C. polytrichus Attems, 1903) [Titova, 1975], Geophilus proximus C.L. Koch, 1847 and Arctogeophilus macrocephalus Folkmanova et Dobroruka, 1960 [Zalesskaja et al., 1982].

\section{Material and methods}

The material for this paper was collected in 20042016 in different places and habitats, largely within the Rostov-on-Don Region. Specimens were taken by handcollecting and pitfall trapping. The material is shared between the collections of the Zoological Museum of the North Caucasian Federal University, Stavropol (ZMS), of the Zoological Museum of the State University of Moscow, Russia (ZMUM) and of the private collection of the second author (AE). Photographs were taken with a DCM 500 digital camera and a Levenhuk DTX 90 digital microscope, images were processed using Adobe Photoshop CS5 software.

Taxonomic part

$$
\begin{gathered}
\text { ORDER SCUTIGEROMORPHA } \\
\text { Family SCUTIGERIDAE } \\
\text { Scutigera coleoptrata (Linnaeus, 1758) } \\
\text { Map } 1 .
\end{gathered}
$$

MATERIAL. 1 ex. (AE), Rostov-on-Don City, in house, 3.VI.2006; 2 ex. (AE), Bokovskiy Distr., Bokovskaya, in house, 
23.IX.2007; 2 ex. (AE), Orlovskiy Distr., Volochaevskiy, 30.IV.2006; 3 ex. (AE), Myasnikovskiy Distr., near Nedvigovka, under stones, 13.IV.2009, all leg. A.P.Evsyukov.

REMARKS. A cosmopolitan polyzonal species [Farzalieva, 2008], earlier recorded from the region by Minoranskiy [1977]. A synanthrope, found in villages and cities.

\section{ORDER LITHOBIOMORPHA \\ Family HENICOPIDAE}

Lamyctes (Lamyctes) emarginatus (Newport, 1844) Map 1.

REMARKS. A multiregional species [Farzalieva, Esyunin, 2008]. In the Rostov-on-on Region, previously recorded near Tarasovka, Tarasovskiy Distr. [Minoranskiy, 1975, 1977].

\section{Family LITHOBIIDAE}

Hessebius multicalcaratus Folkmanova, 1958 Map 1.

REMARKS. Originally described from near KamenskShakhtinskiy, Rostov-on-Don Region and at Lake ArshanZelmen, Republic of Kalmykia [Folkmanova, 1958], this species has since been recorded in the Dnepropetrovsk Region of Ukraine [Zalesskaja, 1978; Farzalieva, 2009; Kunah, 2013]. Previously considered endemic to the Russian Plain [Zalesskaja, Golovatch, 1996], but, according to Farzalieva (in litt.), it occurs also in the middle Urals and western Siberia, Russia.

\section{Lithobius (Lithobius) forficatus (Linnaeus, 1758) Map 1.}

MATERIAL. O7 (ZMS), Rostov-on-Don City, 14.IV.2010; $\sigma^{7}$ (ZMS), Rostov-on-Don City, Botanical Garden, 29.IV.2010, all leg. E.A. Bortnikov; $2 O^{7} \sigma^{7}, 3$ OPO (ZMUM), Rostov-on-Don City, floodplain creek, 14.V.2007; $\sigma^{7}$ (ZMS), same locality, near airport, grove, 20.IV.2008; $2 \mathrm{O}^{7} \sigma^{7}, 3$ 우 (ZMS), same locality, Zelyonyi

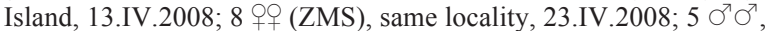
2 우 (ZMS), Tarasovskiy Distr., near Aleksandrovka, Gorodishchenskiy Forestry, floodplain of Kalitva River, 27.VII.2006, all leg. A.Y. Rudaykov; + (ZMS), Salskiy Distr., near Salsk, under rotten straw, 3.V.2004; Ơ (ZMS), Ust'-Donetskiy Distr., near Razdorskaya, 17.VII.2006, all leg. N.O. Morozova; + (ZMS), Azovskiy Distr., near Azov, leaf litter, 15.X.2009, leg. A.V. Dobrinov; ㅇ (ZMS), Verkhnedonskoy Distr., near Kazanskaya, forest belt, 19.VIII.2005, leg. A. Anistratov; $\sigma^{7}$ (ZMS), Zernogradskiy Distr., near Zernograd, forest belt, 3.V.2008; $4 \mathrm{O}^{7} \mathrm{O}^{\prime}, 4$ 우, 2 juv. (ZMUM), Orlovskiy Distr., Rostovskiy Nature Reserve, $10 \mathrm{~km} \mathrm{SW}$ of Strepetov, steppe, 1.V.2005; 5 90 (ZMS), same locality, island, 1.V.2008; $2 O^{7} O^{7}$ (ZMS), near Volochaevskiy, along road, under stones, 30.IV.2006; $2 \mathrm{O}^{7} \mathrm{O}^{7}$ (ZMS), same locality, 30.IV.2010, all leg. A.P. Evsyukov \& D.D. Khisametdinova; 9,1 juv. (ZMS), same locality, 23.IV.2004; 5 O $^{7}, 2$ 우 (ZMUM), same locality, 25.IV.2004, all leg. A.P. Evsyukov; $\bigcirc^{7}, \propto+1$ juv. (ZMS), same locality, under stones, 15.IV.2009, leg. A.P. Evsyukov, V.Y.Shmatko \& A.V. Tikhonov; 우
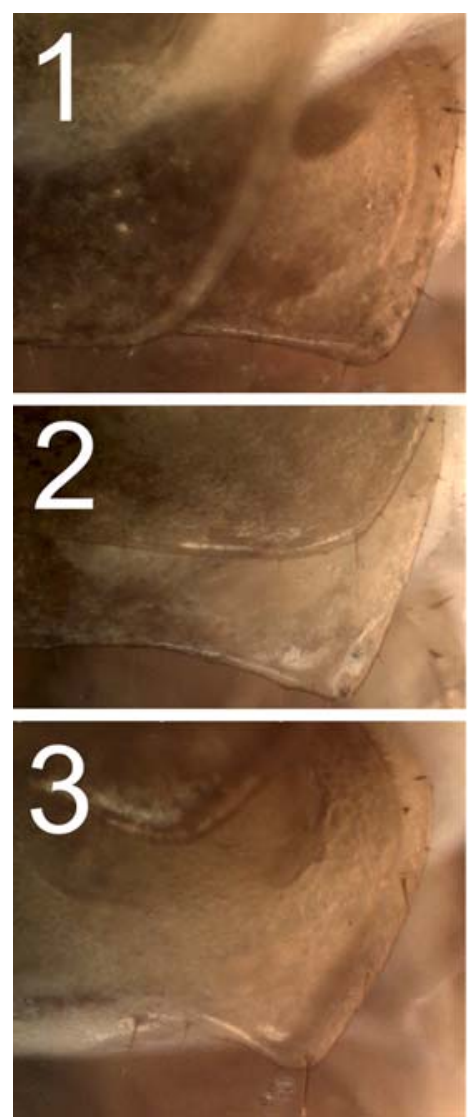
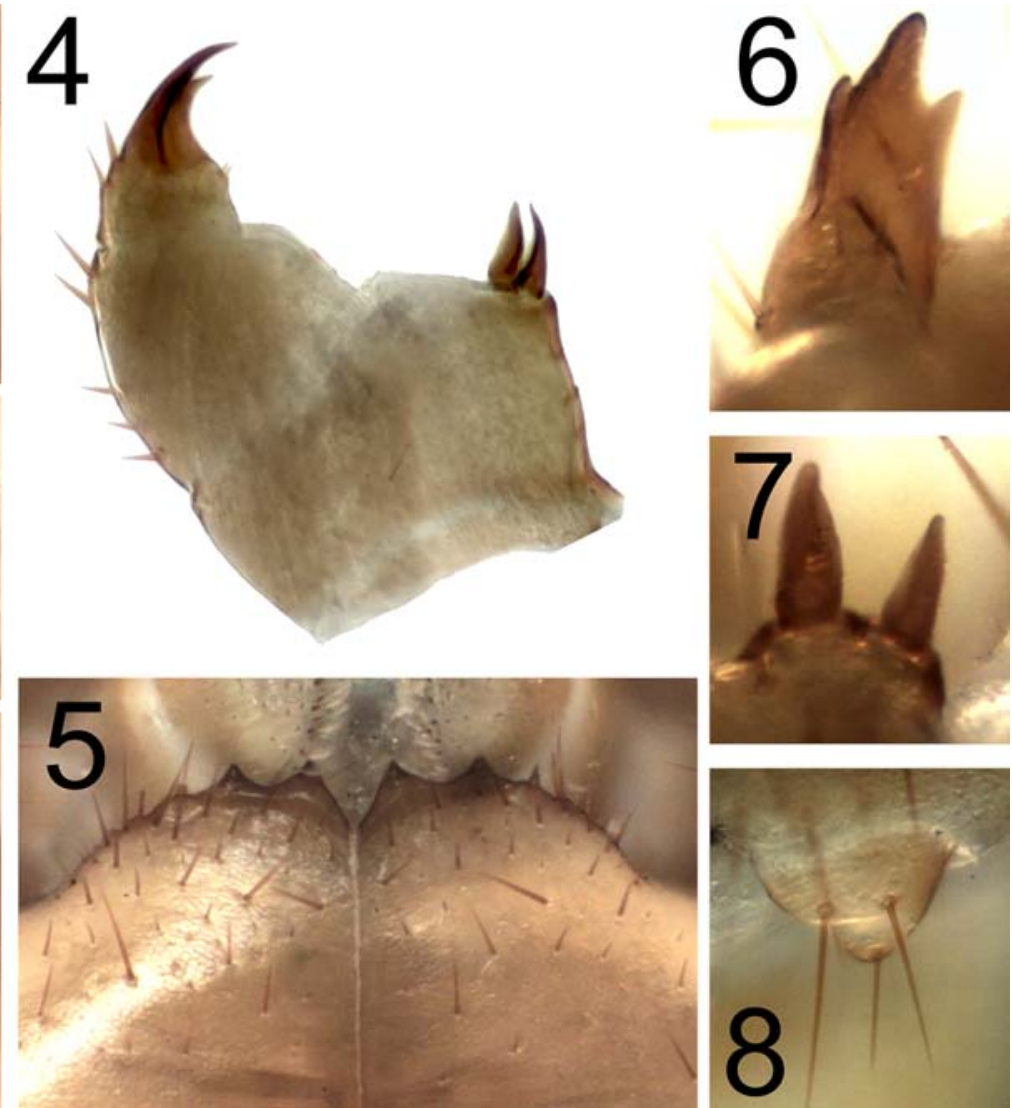

Figs 1-8. Lithobius lucifugus L. Koch, 1862: 1-3 - posterior margin of tergites IX, XI and XIII, respectively; 4 - right female gonopod, mesal view; 5 - coxosternite of forcipular segment; 6 - claw of female gonopod; 7 - spurs of female gonopod, frontal view; 8 - left male gonopod, ventral view. Pictures taken not to scale.

Pис. 1-8. Lithobius lucifugus L. Koch, 1862: 1-3 - задний край соответственно тергитов IX, XI и XIII; 4 - правый гонопод самки, изнутри; 5 - коксостернит ногочелюсти; 6 - коготь гонопода самки; 7 - шпоры гонопода самки, спереди; 8 - левый гонопод самца, снизу. Фотографии без масштаба. 
(ZMS), Millerovskiy Distr. near Olkhovyi Rog, coniferous forest, 24.VII.2006, leg. A.P. Evsyukov; O' (ZMS), Taganrog City, Dubki Park, Quercus grove, 2.VII.2006, leg. D.D. Khisametdinova; 1 juv. (ZMS), Myasnikovskiy Distr., near Nedvigovka, Kamennaya gully, 12.IV.2009, leg. A.P. Evsyukov, D.D. Khisametdinova, D.D.Volkova, I.L.Gorbenko \& V.Y. Shmatko; + (ZMS), same locality, 17.IV.2011, leg. D.D. Khisametdinova \& E.A. Bortnikov.

OTHER MATERIAL. $20^{7} 0^{7}, 2+9$ (ZMS), Republic of Kalmykia, Gorodovikovsk Distr., Gorodovikovsk, Quercus grove, 30.IV.2011, leg. A.P. Evsyukov \& O.A. Borokh.

REMARKS. A multiregional species [Farzalieva, Esyunin, 2008], widespread in the European part of Russia and in the Caucasus, already reported from the Rostov-on-Don Region [Minoranskiy, 1975, 1977; Zalesskaja, 1978; Zalesskaja et al., 1982]. The most abundant species of Lithobiomorpha in our collection.

\section{Lithobius (Lithobius) lucifugus L. Koch, 1862} Figs 1-8, Map 2.

MATERIAL. $40^{7} \sigma^{\top}, 4$ ㅇ (ZMUM), Rostov-on-Don Region, Myasnikovskiy Distr., near Nedvigovka, Kamennaya gully, 12.IV. 2009, leg. A.P. Evsyukov, D.D. Khisametdinova, D.D.Volkova, I.L. Gorbenko \& V.Y. Shmatko; $\sigma^{\top}, 2$ ㅇ (ZMS), Ust'-Donetskiy Distr., near Kanygin, bank of Severskiy Donets River, 14.VI.2009, leg. D.D. Khisametdinova.

REMARKS. A European species [Dányi, 2006], widespread across the European part of Russia, the middle and southern Urals [Zalesskaja, 1978; Zalesskaja et al., 1982; Volkova, 2014a, b; Farzalieva, Esyunin, 2008]. Never previously recorded from the Rostov-on-Don Region.

\section{Lithobius (Lithobius) melanops Newport, 1845}

Figs 9-16, Map 2.

MATERIAL. O7, 9 (ZMUM), Rostov-on-Don City, Botanical Garden, greenhouse, 28.IX.2010, leg. V.Y. Shmatko \& A.V. Malinovkin; $\sigma^{T}$ (ZMS), Yegorlykskiy Distr., near Proshchalnyi, forest belt along road, 18.IV.2008, leg. N.O. Morozova; + (ZMS), Salskiy Distr., near Salsk, forestry, 3.V.2008, leg. A.P. Evsyukov \& D.D. Khisametdinova; + (ZMUM), Ust'-Donetskiy Distr., Nizhnekundryuchenskaya, near Listopadov, dry leaf litter, 24.VII.2010, leg. A.P. Evsyukov.

REMARKS. A European species [Reip, Voigtländer, 2009]. In Russia, known from Karelia, Moscow City [Zalesskaja, 1978; Zalesskaja et al., 1982], the Ulyanovsk Region [Volkova, 2014a], the Penza Region [Volkova, 2015] and the Stavropol Territory [Zuev, 2016]. Apparently, occurring throughout the Holarctic due to anthropochory [Zalesskaja, 1978], mainly inhabiting artificial forest stands. A species new to the regional list.

\section{Lithobius (Monotarsobius) crassipes L. Koch, 1862} Map 2.

MATERIAL. o (ZMS), Rostov-on-Don Region, Salskiy Distr.,

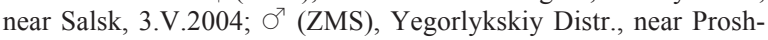
chalnyi, forest belt along road, leaf litter, 18.IV.2008, all leg. N.O. Morozova; 9 (ZMS), Ust'-Donetskiy Distr., Nizhnekundryuchenskaya, near Listopadov, dry leaf litter, 24.VII.2010, leg. A.P. Evsyukov; + (ZMS), near Kanygin, bank of Sukhoi Donets River, leaf litter, 15.VI.2009; + (ZMS), Salskiy Distr., near Manychstroy, bank of Proletarskoe Reservoir, under stones, 16.IV.2009; $\sigma^{2}$, + (AE),
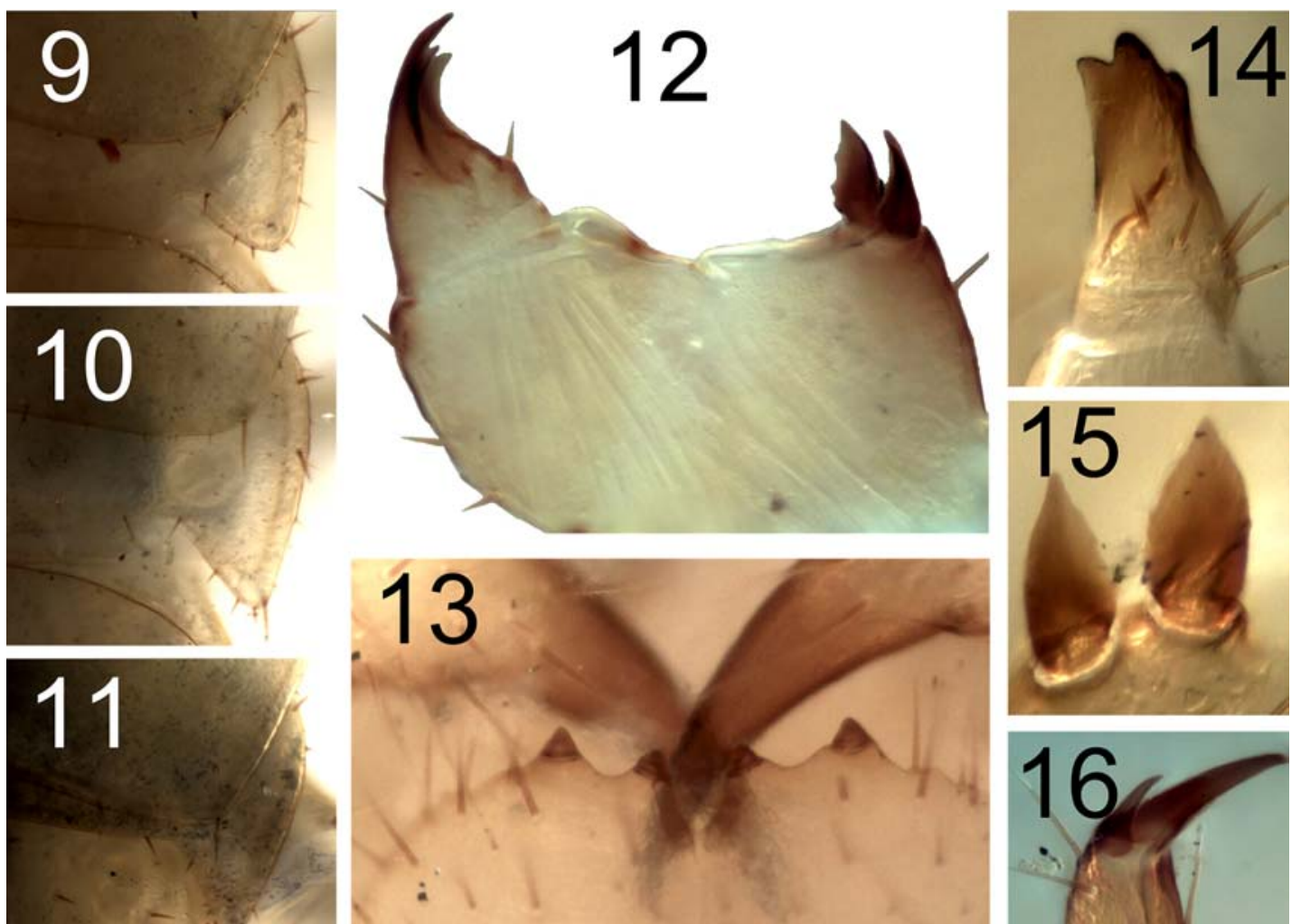

Figs 9-16. Lithobius melanops Newport, 1845: 9-11 — posterior margin of tergites IX, XI and XIII, respectively; 12 - right female gonopod, mesal view; 13 - coxosternite of forcipular segment; 14 - claw of female gonopod; 15 — spurs of female gonopod, frontal view; 16 - pretarsus of leg 15 . Pictures taken not to scale.

Рис. 9-16. Lithobius melanops Newport, 1845: 9-11 — задний край соответственно тергитов IX, XI и XIII; 12 — правый гонопод самки, изнутри; 13 - коксостернит ногочелюсти; 14 - коготь гонопода самки; 15 - шпоры гонопода самки, спереди; 16 - коготь ноги 15. Фотографии без масштаба. 
Orlovskiy Distr., near Volochaevskiy, under stones, 23.IV.2004, det. G. Farzalieva; + (ZMS), same locality, 2.V.2008; O' (ZMS), 10 km SWW of Podgornoye, Mt Lysaya, 2.V.2008; $4 \sigma^{7} \sigma^{7}, 4$, 9 , 3 juv. (ZMUM), same locality, 2.V.2010; $\sigma^{T}$ (AE), Rostovskiy Nature Reserve, near Pravoberezhnyi, bank of Lake Manych-Gudilo, under stones, 21.VII.2004, det. G. Farzalieva; 2 우 (AE), Myasnikovskiy Distr., near Nedvigovka, forest in ravine, 14.IV.2002, det. G. Farzalieva; $O^{7}$,,+ (ZMS), same locality, Kamennaya gully, 5.IV.2005, all leg. A.P. Evsyukov, D.D. Khisametdinova; 3 +o (ZMS), same locality, 12.IV.2009, leg. A.P. Evsyukov, D.D. Khisametdinova, D.D. Volkova, I.L. Gorbenko \& V.Y. Shmatko; ㅇ (ZMS), Neklinovskiy Distr., near Morskoy Chulek, steppe, under stones, 18.IV. 2010, leg. A.P. Evsyukov, D.D. Khisametdinova \& V.Y. Shmatko; $0^{7}, 3$ ㅇ (ZMUM), Kagalnitskiy Distr., near Kagalnitskaya, Pinus forest, 9.IV.2016, leg. R.V. Zuev.

OTHER MATERIAL. $8 \sigma^{7} \sigma^{7}, 8$ 우 (ZMS), Republic of Kalmykia, Gorodovikovskiy Distr., Gorodovikovsk, Quercus grove, 30.IV.2011, leg. A.P. Evsyukov \& O.A. Borokh.

REMARKS. A European species [Reip, Voigtländer, 2009]. In Russia found in Karelia, the Perm Territory, middle Urals, the Moscow and Ulyanovsk regions [Sseliwanoff, 1878; Zalesskaja, 1978; Farzalieva, 2008; Volkova, 2014a] and the Caucasus [Zalesskaja, 1973, 1978]. Previously recorded from the Rostov-on-Don Region [Ghilarov, Folkmanova, 1957; Minoranskiy, 1975, 1977].

Lithobius (Monotarsobius) curtipes C.L. Koch, 1847 Map 2.

MATERIAL. $0^{\top}, 4$ 오 (ZMUM), Rostov-on-Don Region, Sholokhovskiy Distr., near Matveevskiy, 19.VII.2005, leg. E.A. Khachikov; $0^{7}, 1$ juv. (ZMS), Ust'-Donetskiy Distr., near Kanygin, bank of Severskiy Donets River, leaf litter, 15.VI.2009; ㅇ (ZMS), near Olkhovyi, plantations, leaf litter, 16.VI.2009, + (AE), Azovskiy
Distr., delta of Don River, island, 8.VIII.2008, det. G. Farzalieva, all leg. A.P. Evsyukov \& D.D. Khisametdinova; 3 우 (ZMS), Myasnikovskiy Distr., near Nedvigovka, Kamennaya gully, 12.IV.2009, all leg. A.P. Evsyukov, D.D. Khisametdinova, D.D. Volkova, I.L. Gorbenko \& V.Y. Shmatko; $q$ (ZMS), Neklinovskiy Distr., near Morskoy Chulek, steppe, under stones, 18.IV.2010, leg. A.P. Evsyukov, D.D. Khisametdinova \& V.Y. Shmatko; 9 (ZMS), Orlovskiy Distr., Rostovskiy Nature Reserve, under manure, 16.VI.2009, leg. A.P. Evsyukov, D.D. Khisametdinova \& M.A. Tikhonova; $q$ (ZMS), same locality, bank of Lake Lebyazhye, 16.VI.2009, leg. A.P. Evsyukov, V.Y. Shmatko \& A.V. Tikhonov; + (ZMS), Tsimlyanskiy Distr., near Tsimlyansk, Donskoi Nature Park, island, leaf litter, 10.X.2008 leg. N.I. Bulisheva.

REMARKS. A Euro-Siberian species [Farzalieva, Esyunin, 2008]. Previously recorded from the Rostov-on-Don Region [Ghilarov, Folkmanova, 1957; Minoranskiy, 1975, 1977; Zalesskaja, 1978].

\section{Lithobius (Monotarsobius) sseliwanoffi}

(Garbowski, 1897)

Figs 17-20, Map 2.

MATERIAL. $4 \sigma^{7} \sigma^{7}$, + (ZMS), Rostov-on-Don Region, Kagalnitskiy Distr., near Kagalnitskaya, Pinus forest, under burnt logs, 9.IV.2016, leg. R.V. Zuev.

REMARKS. A southern European species [Zalesskaja, Golovatch, 1996], previously recorded in the Rostov-on-Don Region and referred to as Monotarsobius sseliwanoffi [Zalesskaja, 1978; Zalesskaja et al., 1982]. Eason [1997] synonymized L. sseliwanoffi with L. ferganensis (Trotzina, 1894), but this opinion was questioned by Farzalieva [2006]. As noted by Minoranskiy [1977], this species is the most abundant Lithobiomorpha in the region.

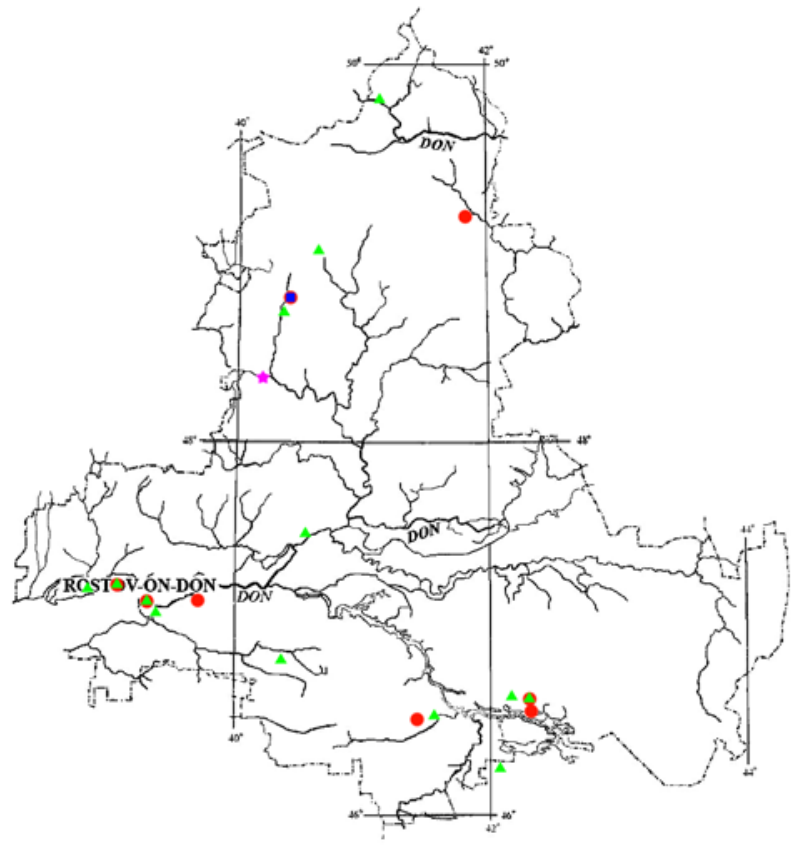

Map 1. Distribution of centipedes in the Rostov-on-Don Region red circle - Scutigera coleoptrata; blue square - Lamyctes emarginatus; purple star — Hessebius multicalcaratus; green triangle Lithobius forficatus.

Карта 1.Распространение губоногих многоножек на территории Ростовской области: красный круг - Scutigera coleoptrata; синий квадрат - Lamyctes emarginatus; фиолетовая звезда - Hessebius multicalcaratus; зеленый треугольник — Lithobius forficatus.

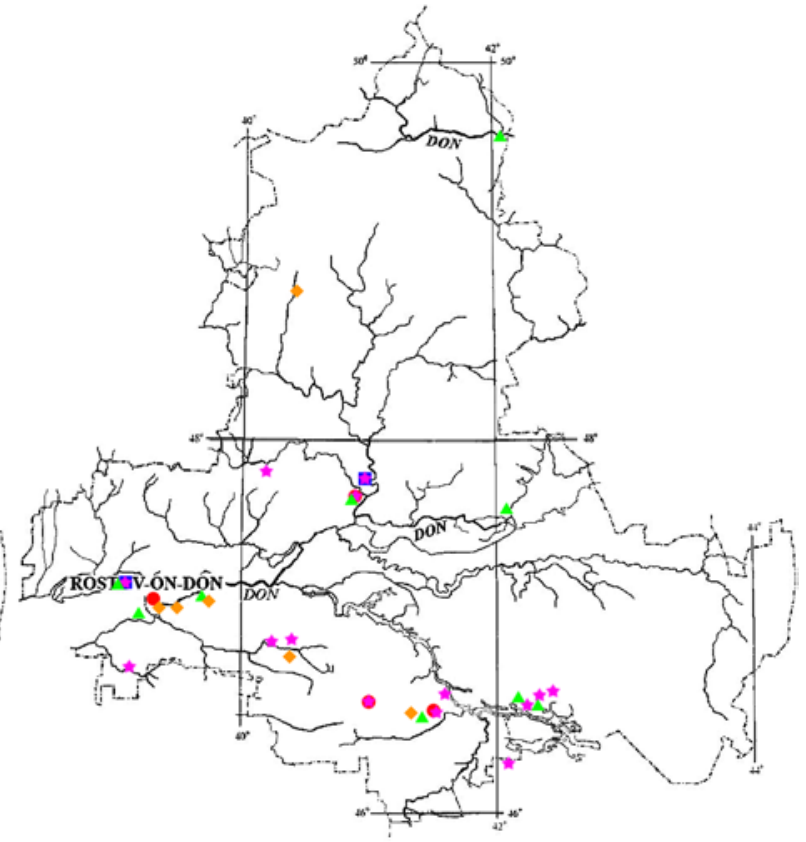

Map 2. Distribution of centipedes in the Rostov-on-Don Region: red circle - Lithobius melanops; blue square - L. lucifugus; purple star - L. crassipes; green triangle — L. curtipes; brown diamond L. ferganensis.

Карта 2. Распространение губоногих многоножек на территории Ростовской области: красный круг — Lithobius melanops; синий квадрат - L. lucifugus; фиолетовая звезда - L. crassipes; зеленый треугольник - L. curtipes; коричневый ромб - L. ferganensis. 


\section{ORDER SCOLOPENDROMORPHA \\ Family SCOLOPENDRIDAE}

Scolopendra cingulata Latreille, 1789

MATERIAL. 1 juv. (ZMS), Rostov-on-Don Region, Myasnikovskiy Distr., near Nedvigovka, second terrace above Malyi Donets River floodplain, 10.VIII.2003, leg. A.P. Evsyukov \& D.D. Khisametdinova; 2 juv. (ZMS), near Nedvigovka, Kamennaya gully, under stones, 18.IV.2010, E.A. Bortnikov; + (ZMS), Salskiy Distr. near Salsk, 8.III.2015, leg. A. Strelnik; O' (ZMS), Kagalnitskiy Distr., near Kagalnitskaya, Pinus forest, under burnt logs, 9.IV.2016, leg. R.V. Zuev.

REMARKS. A Mediterranean species [Stoev, 2002], recorded in Russia from the southern European part and from the Caucasus [Kessler, 1876; Lignau, 1903; Zalesskaja, Schileyko, 1991, 1992]. Inhabiting steppe biotopes in the southern parts of the Rostov-on-Don Region [Minoranskiy, 1975, 1977]. Included in the first edition of the Red Data Book of the Rostov-on-Don Region [Minoranskiy, 2004], but, as the numbers had since increased, this species was excluded from the second edition.

\section{Family CRYPTOPIDAE}

Cryptops (Cryptops) anomalans Newport, 1844 Map 3.

MATERIAL. 1 ad. (ZMS), Rostov-on-Don Region, Orlovskiy Distr., Rostovskiy Nature Reserve, bank of Lake Manych-Gudilo, 11.IX.2004, leg. V.A. Minoranskiy; 5 ad. (ZMS), near Volochaevskiy, 23.IV.2004; 8 ad. (ZMUM), same locality, 25.IV.2004, all leg. A.P. Evsyukov; 1 ad. (ZMS), Neklinovskiy Distr., near Morskoy Chulek, steppe, under stones, 18.IV.2010, leg. A.P. Evsyukov, D.D. Khisametdinova \& V.Y. Shmatko; 2 ad. (ZMS), Kagalnitskiy Distr., near Kagalnitskaya, Pinus forest, under burnt logs, 9.IV.2016, leg. R.V. Zuev.

REMARKS. A European species [Stoev, 2002], previously recorded from the Kherson and Lugansk regions of Ukraine, the Crimea and the Rostov-on-Don Region, Russia [Ghilarov, Folkmanova 1957; Minoranskiy, 1977; Zalesskaja, Schileyko, 1991, 1992].

Cryptops (Cryptops) caucasius Verhoeff, 1934 Figs 21-22, Map 3.

MATERIAL. 1 juv. (ZMS), Rostov-on-Don Region, Rostovon-Don City, Botanical Garden, greenhouse, 28.IX.2010, leg. V.Y. Shmatko \& A.V. Malinovkin.
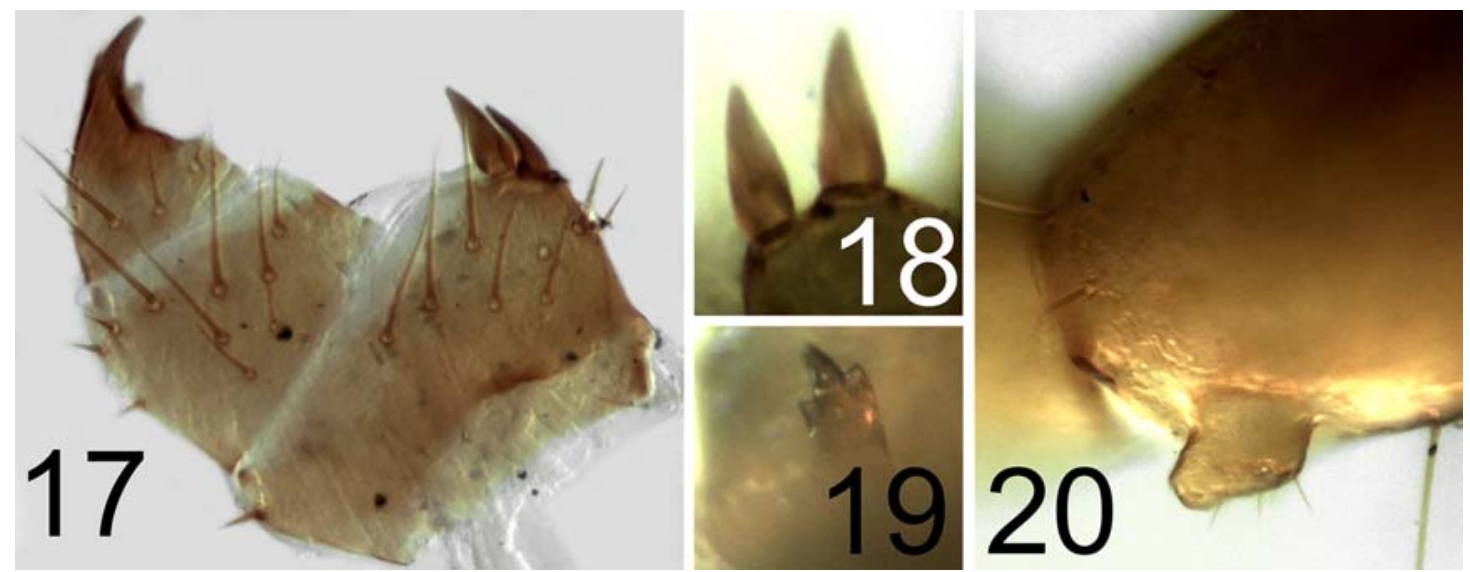

Figs 17-20. Lithobius (Monotarsobius) sseliwanoffi (Garbowski, 1897): 17 - left female gonopod, ventral view; 18 - spurs of female gonopod, frontal view; 19 - claw of female gonopod; 20 - distal part of left tibia $15\left(\sigma^{7}\right)$, lateral view. Pictures taken not to scale.

Рис. 17-20. Lithobius (Monotarsobius) sseliwanoffi (Garbowski, 1897): 17 - левый гонопод самки, изнутри; 18 - шпоры гонопода самки, спереди; 19 - коготь гонопода самки; 20 - дистальная часть голени левой 15 ноги (О'). Фотографии без масштаба. 
promoted to a full species status [Pereira, Hoffman, 1993]. Folkmanova [1956] recorded it from the Veselovskiy, Ust'Donetskiy and Krasnosulinskiy districts of Rostov-on-Don Region and in the Krasnodar Territory, Russia; also from the Lugansk Region of Ukraine [Ghilarov, Folkmanova, 1957].

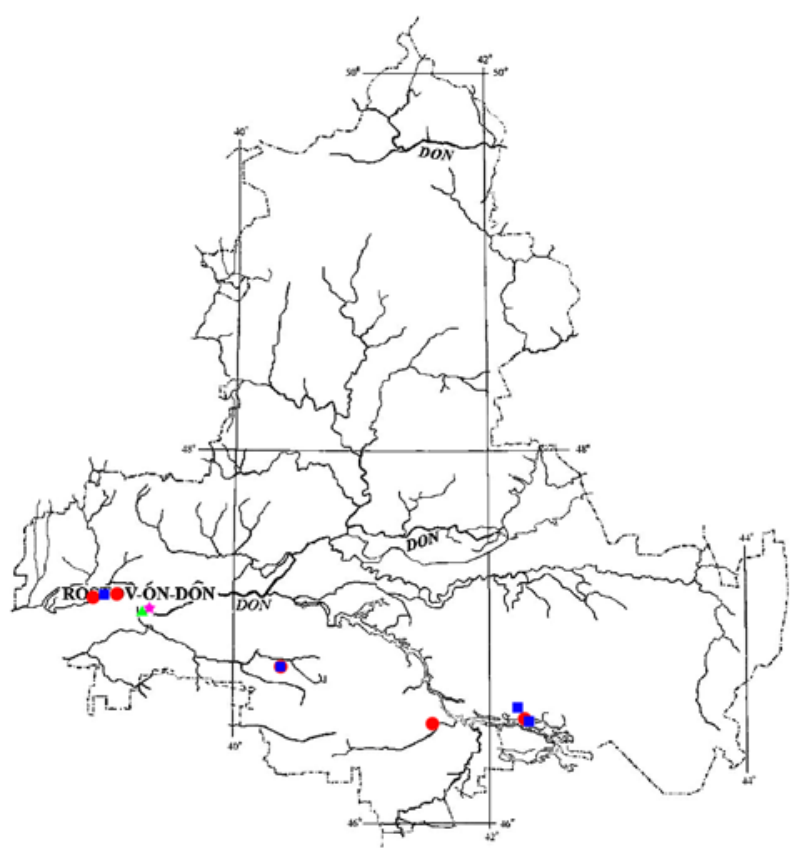

Map 3. Distribution of centipedes in the Rostov-on-Don Region: red circle - Scolopendra cingulata; blue square - Cryptops anomalans; purple star $-C$. caucasius; green triangle $-C$. hortensis.

Карта 3. Распространение губоногих многоножек на территории Ростовской области: красный круг - Scolopendra cingulata; синий квадрат - Cryptops anomalans; фиолетовая звезда - C. caucasius; зеленый треугольник - C. hortensis.
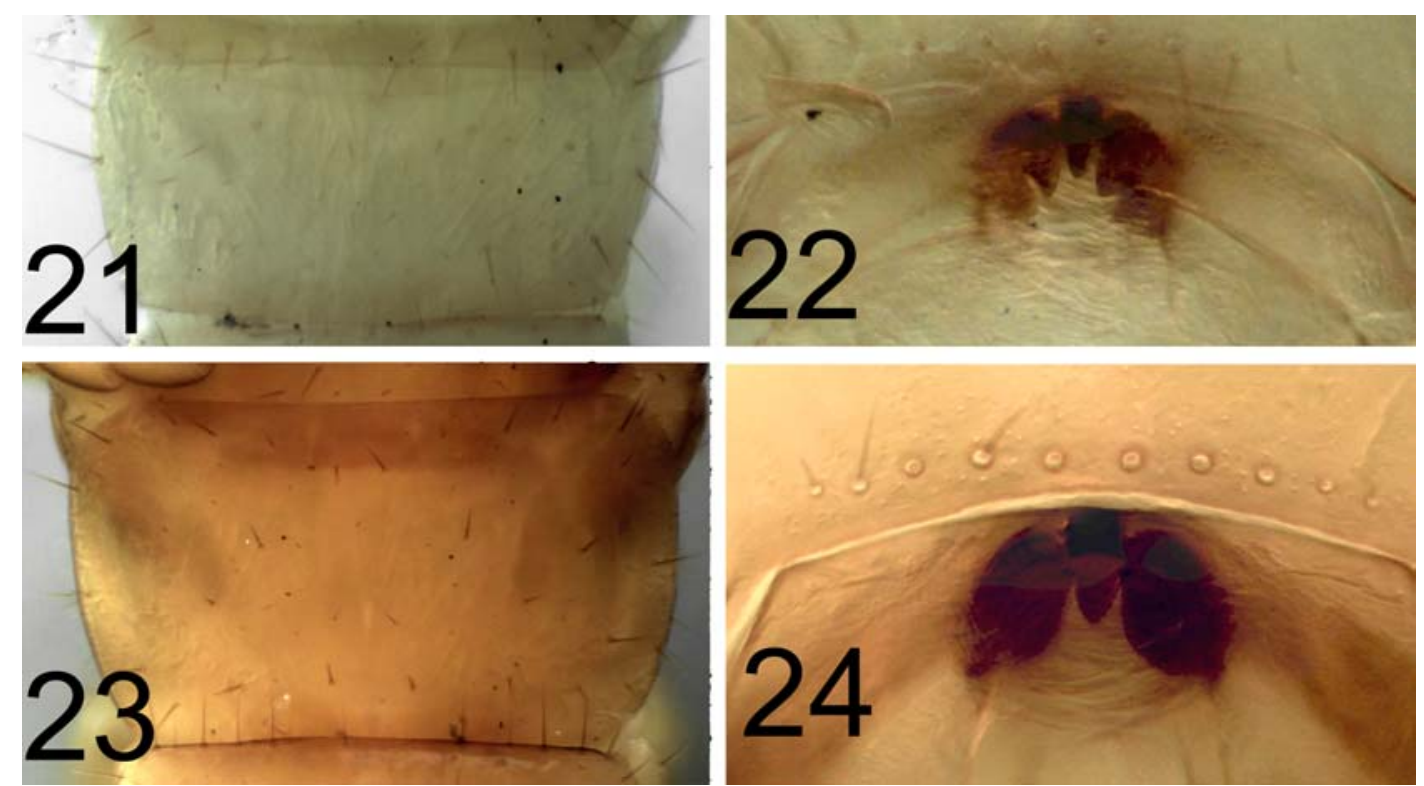

Figs 21-24. Cryptops spp.: 21-22 - C. caucasius Verhoeff, 1934; 23-24 - C. hortensis (Donovan, 1810); 21, 23 - tergite I; 22, 24 labrum. Pictures taken not to scale.

Рис. 21-24. Cryptops spp.: 21-22 - C. caucasius Verhoeff, 1934; 23-24 - C. hortensis (Donovan, 1810); 21 и 23 - тергит I; 22 и 24 - лабрум. Фотографии без масштаба.
Escaryus retusidens Attems, 1904

Map 4.

REMARKS. A Central Asian - Eastern European species, widespread in Moldova, the Crimea, eastern Ukraine,

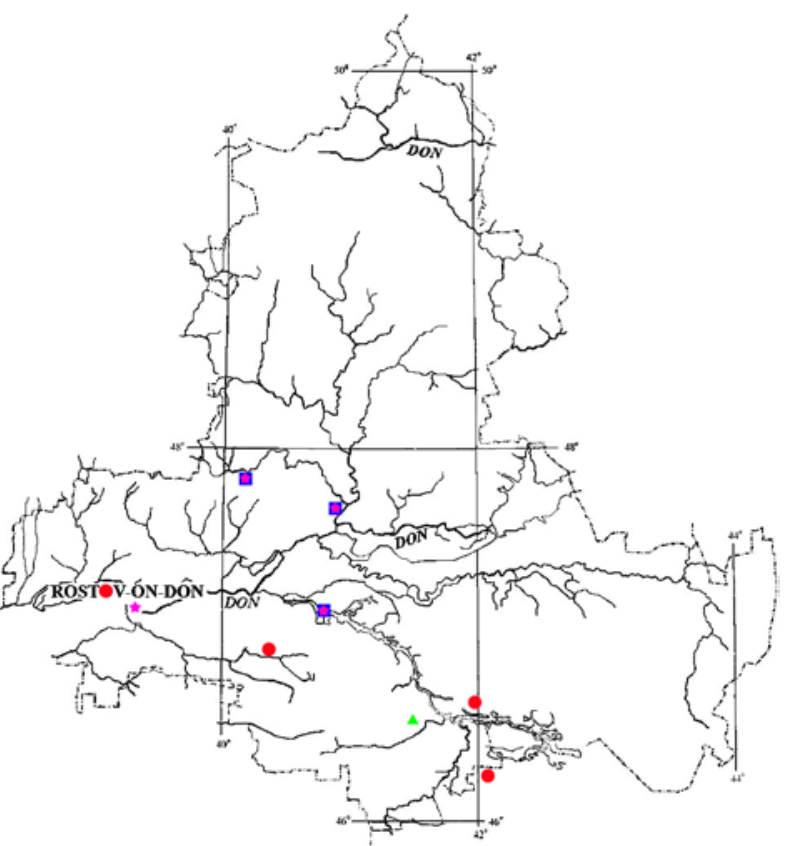

Map 4. Distribution of centipedes in the Rostov-on-Don Region: red circle - Henia bicarinata; blue square - Escaryus ornatus; purple star - E. retusidens; green triangle - Schendyla nemorensis.

Карта 4. Распространение губоногих многоножек на территории Ростовской области: красный круг - Henia bicarinata; синий квадрат - Escaryus ornatus; фиолетовая звезда -E. retusidens; зеленый треугольник — Schendyla nemorensis. 
the Krasnodar, Voronezh and Penza regions of Russia and in Kazakhstan [Titova, 1972; Volkova, 2015]. In the Rostovon-Don Region, it has been recorded in the city of Rostov-onDon, as well as in the Krasnosulinskiy, Ust'-Donetskiy and Tarasovskiy districts [Folkmanova, 1956; Ghilarov, Folkmanova 1957; Minoranskiy, 1977].

\section{Schendyla nemorensis (C.L. Koch, 1837) Map 4.}

REMARKS. A European species [Stoev, 2002]. In Russia, recorded from the Moscow, Kursk, Belgorod, Rostovon-Don [Minoranskiy, 1975, 1977; Zalesskaja et al., 1982] and Stavropol regions [Zuev, 2016].

\section{Family GEOPHILIDAE}

\section{Arctogeophilus macrocephalus Folkmanova et Do- broruka, 1960}

REMARKS. A trans-Eurasian boreal species [Farzalieva, 2008], originally described from Tatarstan [Folkmanova, Dobroruka, 1960]. Widespread from Transcarpathia to Chukot Peninsula and Sakhalin Island. It has already been recorded from the Rostov-on-Don Region [Zalesskaja et al., 1982]. In the European part of Russia, this species inhabits the belts of forested steppe and deciduous forest [Zalesskaja et al., 1982].

\section{Clinopodes caucasicus (Sseliwanoff, 1884)} Figs 25-28, Map 5.

MATERIAL. 1 juv. (ZMS), Rostov-on-Don Region, Salskiy Distr., near Salsk, forestry, 30.IV.2008, leg. A.P. Evsyukov \& D.D. Khisametdinova; 9 (ZMUM), Kagalnitskiy Distr., near Kagalnitskaya, Pinus forest, under burnt logs, 9.IV.2016, leg. R.V. Zuev.

OTHER MATERIAL. $2 \sigma^{7} \sigma^{7}, 2$ OO (ZMS), Republic of Kalmykia, Gorodovikovskiy Distr., Gorodovikovsk, Quercus grove, 30.IV.2011, leg. A.P. Evsyukov \& O.A. Borokh.

REMARKS. Basically a Caucasian species, widespread across the Caucasus, also recorded from Anatolia [Sseliwanoff, 1883; Muralewicz, 1907; Bonato et al., 2011]. Above seem to be the northernmost records of this species. Apparently, this forest species gets into the steppe zone through forest belts. In the Rostov-on-Don Region and the Republic of Kalmykia, C. caucasicus occurs only south of the KumaManych Depression.

\section{Clinopodes flavidus C.L. Koch, 1847 Map 5.}

MATERIAL. O (ZMS), Rostov-on-Don City, 14.IV.2010, leg. E.A. Bortnikov.

REMARKS. A Turano-Euro-Mediterranean species [Zapparoli, 1999], in Russia recorded from the Crimea, Caucasus and Penza Region [Sseliwanoff, 1883; Lignau, 1903; Attems, 1907; Volkova, 2015]. Recorded by Titova [1975] from the Crimea, the Caucasus, the Republic of Kalmykia and the Rostov-on-Don Region, referred to as C. polytrichus Attems, 1903.

In a recent paper [Zuev, 2016], C. flavidus was erroneously reported from the Stavropol Territory. Actually, the pertinent material belongs to C. escherichii (Verhoeff, 1896).

\section{Clinopodes escherichii (Verhoeff, 1896) \\ Map 5.}

MATERIAL. 2 ㅇ (ZMS), Rostov-on-Don Region, Yegorlykskiy Distr., near Proshchalnyi, forest belt along road, leaf litter, 18.IV.2008; $4 O^{7} \sigma^{7}, 2$ 우, 2 juv. (ZMUM), Salskiy Distr., near Salsk, 3.V.2004, all leg. N.O. Morozova; O' (ZMS), same locality, forestry, 3.V.2008; + (ZMS), Myasnikovskiy Distr., near Nedvigovka, delta of Don River, island, 27.IV.2007, all leg. A.P. Evsyukov \& D.D. Khisametdinova; 2 우 (ZMS), Kamennaya gully, 12.IV.2009, leg. A.P. Evsyukov, D.D.Khisametdinova, D.D. Volkova, I.L. Gorbenko \& V.Y. Shmatko; + (ZMS), Orlovskiy Distr., Volochaevskiy, under shelters, 23.IV.2004, leg. A.P. Evsyukov; $O^{7}$ (ZMS), Rostovskiy Nature Reserve, Lake Lebyazhye, steppe, under manure, 15.IV.2009, leg. A.P. Evsyukov, D.D. Khisametdinova \& M.A. Tikhonova.

OTHER MATERIAL. $20^{7} \sigma^{7}, 2$ OP (ZMS), Republic of Kalmykia, Gorodovikovskiy Distr., Gorodovikovsk, Quercus grove, 30.IV.2011, leg. A.P. Evsyukov \& O.A. Borokh; 9 (ZMS), Astrakhan Region, Limanskiy Distr., near Yandyki, 20.IV.2011, leg. I.L. Gorbenko.

REMARKS. An eastern Mediterranean species [Simaiakis et al., 2013]. In the ex-USSR, it was recorded in Moldova, Transcarpathia, the Crimea and Middle Asia [Attems, 1901, 1907; Titova, 1975]. According to Ghilarov et Folkmanova [1957], Titova [1975] and Minoranskiy [1977], in the Rostov-on-Don Region C. escherichii occurs near the Manych River in the southern part of the region, never recorded north of the Don River.
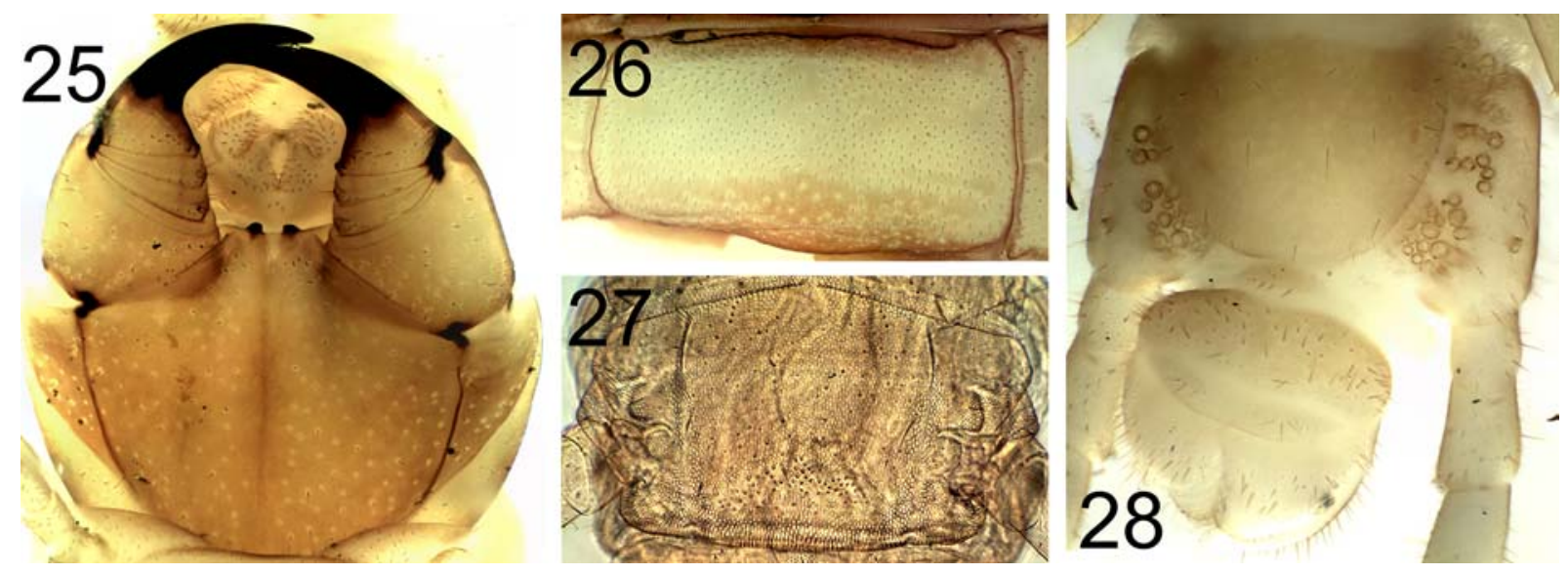

Figs 25-28. Clinopodes caucasicus (Sseliwanoff, 1883). 25 - forcipular segment, ventral view; 26 — anterior leg-bearing sternite; 27 posterior leg-bearing sternite; 28 - ultimate leg-bearing segment, ventral view. Pictures taken not to scale.

Рис. 25-28. Clinopodes caucasicus (Sseliwanoff, 1883). 25 - ногочелюсть, снизу; 26 - передний стернит; 27 - задний стернит; 28 - сегмент конечных ног, снизу. Фотографии без масштаба. 


\section{Diphyonyx sukacevi (Folkmanova, 1956) Map 5.}

REMARKS. A Caucasian species, originally described as Brachygeophilus sukacevi from near Erivanskaya, Krasnodar Territory and in Manychskiy Forestry, Rostov-on-Don Region [Folkmanova, 1956]. Minoranskiy [1977] found this species near the city of Rostov-on-Don and at Gigant, Salskiy Distr. This centipede seems to be confined to the southern parts of the region.

\section{Diphyonyx garutti (Folkmanova et Dobroruka, 1960)}

REMARKS. A Caucasian species, originally described from the Krasnodar Territory as Brachygeophilus sukacevi garutti. Minoranskiy [1977] recorded D. garutti at Manych, but that seems to have been a misidentification of $D$. conjungens (Verhoeff, 1898). Both species are indeed very similar. The main difference lies is structure of the labrum: 1-2 tubercles and 2-4 slender filaments in $D$. conjungens, versus 2-4 slender filaments in D. garutti [Bonato et al, 2008]. Since $D$. conjungens has been found in the Stavropol Territory, Adygea and the Crimea [Attems, 1929; Zuev, 2016], the occurrence of this species also in the Rostov-on-Don Region is highly probable.

\section{Geophilus flavus (De Geer, 1778) Map 6.}

MATERIAL. 1 juv. (ZMS), Rostov-on-Don City, Botanical Garden, 29.IV.2010, leg. E.A. Bortnikov; 1 juv. (ZMS), Myasnikovskiy Distr., near Nedvigovka, forest, 14.IV.2002, leg. A.P. Evsyukov \& D.D. Khisametdinova; 9 (ZMS), Kamennaya gully, 12.IX.2009, leg. A.P.Evsyukov, D.D. Khisametdinova, D.D. Volkova, I.L. Gorbenko \& V.Y. Shmatko.

REMARKS. A Euro-Siberian species [Stoev, 2002], found in the Kamenskiy and Ust'-Donetskiy districts of Rostov-onDon Region, referred to as Schizotaenia tristanica (Attems,

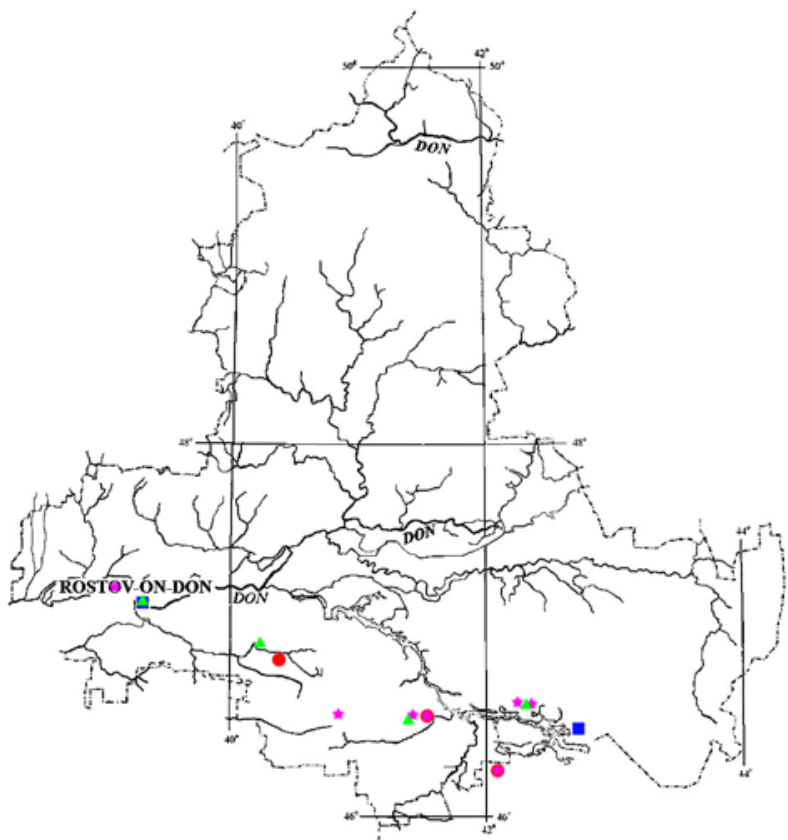

Map 5. Distribution of centipedes in the Rostov-on-Don Region: red circle - Clinopodes caucasicus; blue square - C. flavidus; purple star - C. escherichii; green triangle — Diphyonyx sukacevi.

Карта 5. Распространение губоногих многоножек на территории Ростовской области: красный круг - Clinopodes caucasicus; синий квадрат - C. flavidus; фиолетовая звезда - $C$. escherichii; зеленый треугольник — Diphyonyx sukacevi.
1928) [Ghilarov, Folkmanova 1957; Minoranskiy, 1975, 1977], S. ornata Folkmanova, Dobroruka, 1960 [Folkmanova, Dobroruka, 1960; Minoranskiy, 1975, 1977] or S. longicornis (Leach, 1815) [Minoranskiy, 1975, 1977]. All these names are junior synonyms of G. flavus [Bonato, Minelli, 2014].

Geophilus proximus C.L. Koch, 1847

REMARKS. A European species [Stoev, 2002], widespread in the European part of Russia, also recorded from western Siberia and the Urals [Sseliwanoff, 1883; Farzalieva, 2008; Ruchin, 2014; Volkova, 2014b, 2015]. Reported from the Rostov-on-Don Region as well, but without a precise locality [Zalesskaja et al., 1982]. Therefore, the distribution of $G$. proximus in the region still remains to be clarified.

\section{Pachymerium ferrugineum (C.L. Koch, 1835) Map 6.}

MATERIAL. $2 \sigma^{7} \sigma^{7}, 6$ oᄋ (ZMUM), Rostov-on-Don City, Ostrovskogo Park, 23.IV.2007; $2 \sigma^{7} \sigma^{7}, 2$ 오 (ZMS), Zelyonyi

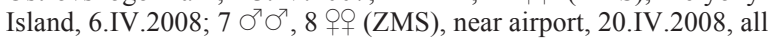
leg. A.Y. Rudaykov; O (ZMS), Neklinovskiy Distr., near Grecheskie Roty, bank of Mius River, 2.V.2009, leg. I.L. Gorbenko; O', + (ZMUM), Ust'-Donetskiy Distr., near Kanygin, Medvezhya gully, 8.VII.2008, leg. A.V. Tikhonov; ㅇ (ZMS), Nizhnekundryuchenskaya, near Ogib, 24.VII.2010, leg. D.D. Khisametdinova; O' (ZMS), near Olkhovyi, plantations, 16.IV.2009; 3 ㅇ (ZMS), Myasnikovskiy Distr., near Nedvigovka, 5.IV.2005, all leg. A.P. Evsyukov \& D.D. Khisametdinova; $3 \mathrm{O}^{7} \mathrm{O}^{7}$, $q$ (ZMS), Kamennaya gully, 12.IV. 2009, leg. A.P. Evsyukov, D.D.Khisametdinova, D.D. Volkova, I.L. Gorbenko \& V.Y. Shmatko; + (ZMS), Orlovskiy Distr., Volochaevskiy, 25.IV.2004, leg. A.P. Evsyukov; $0^{7}$ (ZMS), Rostovskiy Nature Reserve, near Manych, shore of Lake Gruzskoe, 25.VI.2005; 오 (ZMS), $10 \mathrm{~km} \mathrm{SW}$ of Strepetov, 1.V.2005; O $\mathrm{O}^{7}, 5$ 우 (ZMS), $10 \mathrm{~km}$ SWW of Podgornoye, Mt Lysaya, 2.V.2010, all leg. A.P. Evsyukov $\&$ D.D. Khisametdinova; + (ZMS), Lake Lebyazhye, steppe, under manure, 15.IV.2009, leg. A.P. Evsyukov, D.D. Khisametdinova \& M.A. Tikhonova; 3 of (ZMUM), Rostovskiy Nature Reserve, 15.IV.

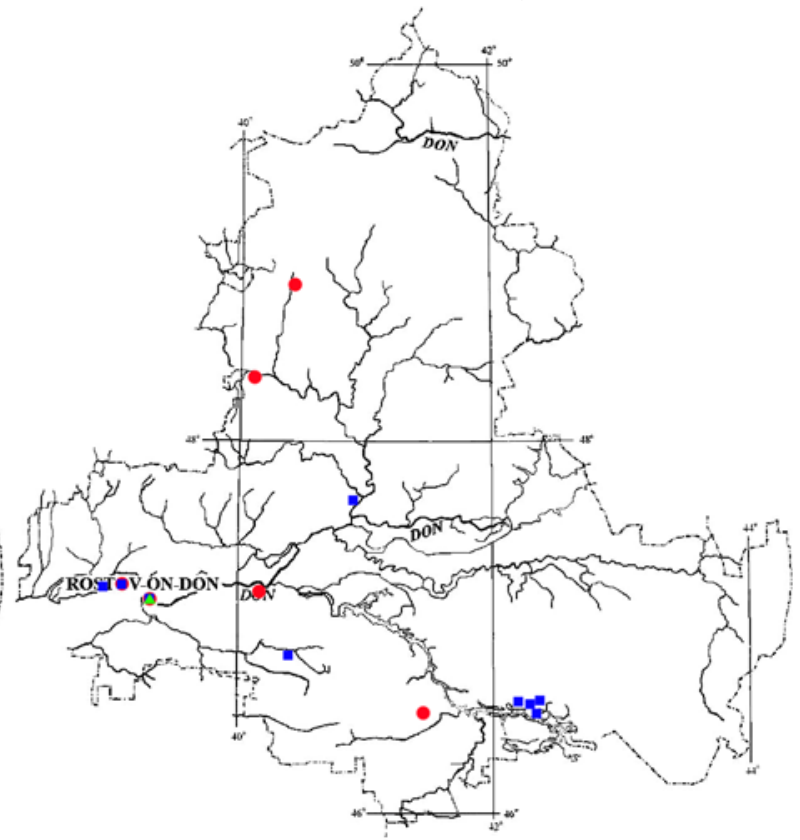

Map 6. Distribution of centipedes in the Rostov-on-Don Region: red circle — Geophilus flavus; blue square - Pachymerium ferrugineum; green triangle - Strigamia cf transsilvanica.

Карта 6. Распространение губоногих многоножек на территории Ростовской области: красный круг — Geophilus flavus; синий квадрат - Pachymerium ferrugineum; зеленый треугольник - Strigamia $\mathrm{cf}$ transsilvanica. 
2009, leg. A.P.Evsyukov, D.D. Khisametdinova, V.Y. Shmatko \& A.V. Tikhonov; 3 juv. (ZMS), Kagalnitskiy Distr., near Kagalnitskaya, Pinus forest, 9.IV.2016, leg.R.V.Zuev.

REMARKS. A trans-Palaearctic species [Farzalieva, 2008], widespread across Russia [Zalesskaja et al., 1982], recorded also from the Rostov-on-Don Region [Ghilarov, Folkmanova 1957]. Minoranskiy [1977] noted that this species inhabits all of the main biotopes in the region, including saline soils.

\section{Family LINOTAENIIDAE}

Strigamia cf. transsilvanica (Verhoeff, 1928)

$$
\text { Figs 29-32, Map } 6 .
$$

MATERIAL. $\sigma^{7}$ (ZMUM), Rostov-on-Don City, shore of Rostovskoe More Reservoir, 24.VI.2012; 2 우 (ZMS), same locality, 24.VI.2012, all leg. A.V. Gorovtsov.

REMARKS. An eastern and southern European species [Reip, Voigtländer, 2009]. Above are its first records from Russia. This species is widespread in Europe, ranging from the Alps in the west to the Carpathians in the east, and from Baltia in the north to Greece in the south [Bonato et al., 2012].

\section{Conclusions}

As a result of our studies, the centipede fauna of the Rostov-on-Don Region is found to include at least 27 species, six of which are recorded from the region for the first time, including one new to the fauna of Russia:
Strigamia cf. transsilvanica. Species with a European distribution pattern (six) dominate the fauna. Caucasian species (Clinopodes caucasicus, Cryptops caucasius and Diphyonyx sukacevi) occur only in the southern areas of the region. Apparently, their distributions are limited by the Kuma-Manych Depression from the north. Another two species (Scutigera coleoptrata and Lamyctes emarginatus) are only synanthropic in distribution. Future faunistic studies on the centipedes of the Rostov-on-Don Region and adjacent areas will reveal further records.

ACKNOWLEDGEMENTS. We thank all colleagues who rendered material for the present study. We are also deeply grateful to Arkady A. Schileyko (Moscow, Russia) and G.Sh. Farzalieva (Perm, Russia) for the help in determining certain material and to S.I. Golovatch (Moscow, Russia) for providing much of the relevant literature and assisting in the present publication. G.Sh. Farzalieva and J.S. Volkova (Ulyanovsk, Russia) made several important corrections and suggestions to advanced drafts.

\section{References}

Attems C.G. 1901. Myriopoden // Zoologische Ergebnisse der dritten asiatischen Forschungsreise des Grafen Eugen Zichy. S.275-310. Attems C.G. 1907. Myriopoden aus der Krim und dem Kaukasus, von Dr. A. Stuxberg gesammelt // Arkif för Zoologi. Bd.3. No.25. S.1-16.
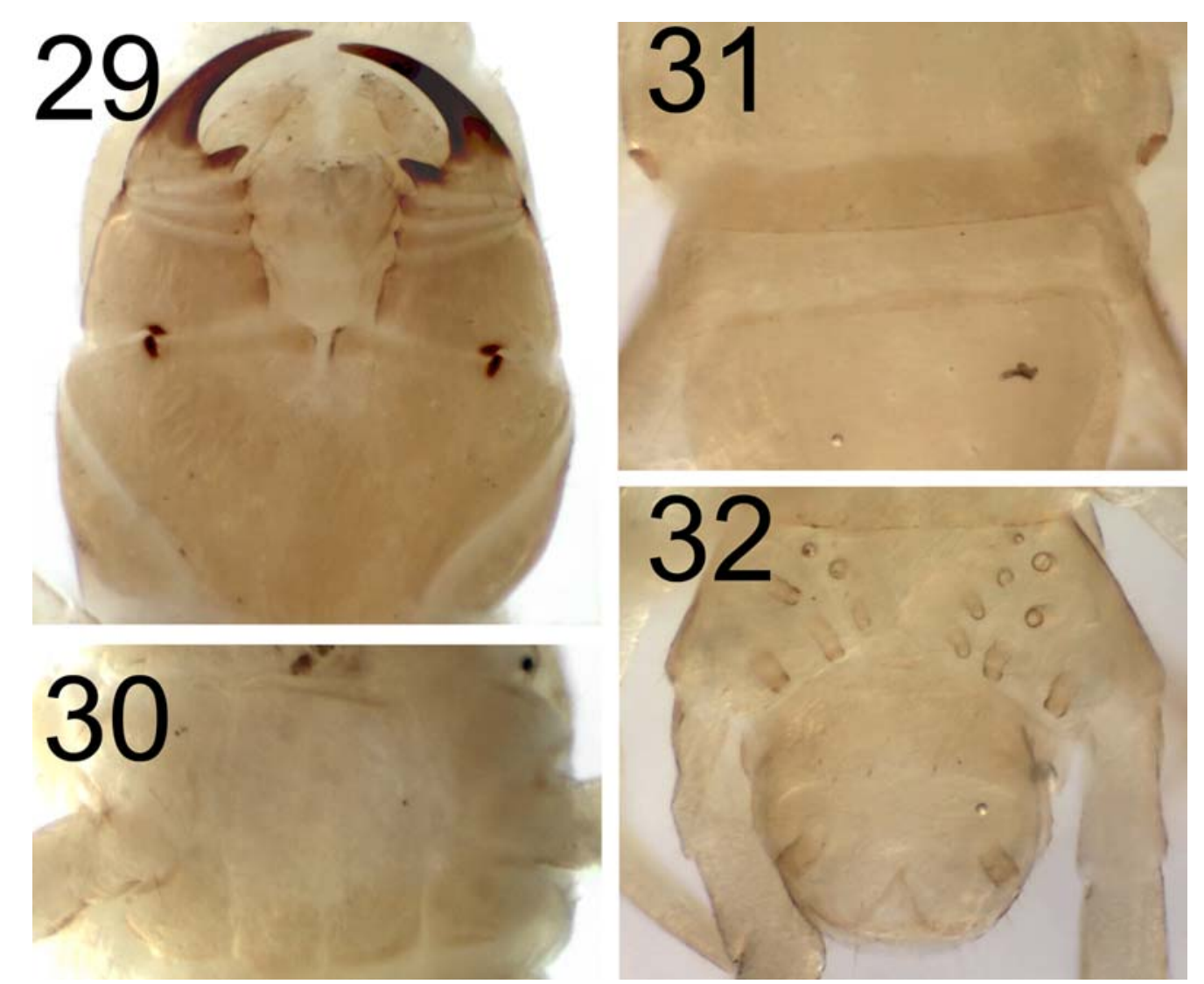

Figs 29-32. Strigamia cf. transsilvanica (Verhoeff, 1928): 29 - forcipular segment, ventral view; 30 - sternite of anterior leg-bearing segment; 31 - ultimate pleuropretergite; 32 - caudal part of body (+), ventral view. Pictures taken not to scale.

Рис. 29-32. Strigamia cf. transsilvanica (Verhoeff, 1928): 29 — ногочелюсть, снизу; 30 - передний стернит; 31 - плевропретергит конечных ног; 32 - задний конец тела (+), снизу. Фотографии без масштаба. 
Attems C.G. 1929. Myriapoda. 1. Geophilomorpha // Das Tierreich. Lfg.52. S.1-388.

Bonato L., Zapparoli M., Minelli A. 2008. Morphology, taxonomy and distribution of Diphyonyx gen. n., a lineage of geophilid centipedes with unusually shaped claws (Chilopoda: Geophilidae) // European Journal of Entomology. Vol.105. P.343-354

Bonato L., Iorio É., Minelli A. 2011. The centipede genus Clinopodes C. L. Koch, 1847 (Chilopoda, Geophilomorpha, Geophilidae): reassessment of species diversity and distribution, with a new species from the Maritime Alps (France) // Zoosystema. Vol.33. No.2. P.175-205.

Bonato L., Dányi L., Socci A.A., Minelli A. 2012. Species diversity of Strigamia Gray, 1843 (Chilopoda: Linotaeniidae): a preliminary synthesis // Zootaxa. Vol.3593. P.1-39.

Bonato L., Minelli A. 2014. Chilopoda Geophilomorpha of Europe: a revised list of species, with taxonomic and nomenclatorial notes // Zootaxa. Vol.3770. No.1. P.1-136.

Dányi L. 2006. Contribution to the Chilopoda fauna of the Maramures (Romania) // Studia Universitatis "Vasile Goldis". Seria Stiintele Vietii (Life Sciences Series). T.17. P.43-46.

Dányi L. 2008. Review and contribution to the Chilopoda fauna of Maramures (Romania) // Studia Universitatis "Vasile Goldis". Seria Stiintele Vietii (Life Sciences Ser.). T.18. Suppl. P.185197.

Eason E.H. 1997. On some Lithobiomorpha from the mountains of Kirghizia and Kazakhstan (Chilopoda) // Arthropoda Selecta. Vol.6. Nos 1-2. P.117-121

Farzalieva G.Sh. 2006. New species of the lithobiid genus Lithobius (Monotarsobius) (Chilopoda: Lithobiomorpha: Lithobiidae) from eastern Kazakhstan // Arthropoda Selecta. Vol.15. No.2. P. 99-117.

Farzalieva G.Sh. 2008. [Fauna and chorology of myriapods (Myriapoda) of the Urals and Cisuralia] // PhD Thesis (Kandidat. dissert.) Moscow. 189 pp. [in Russian].

Farzalieva G.Sh. 2009. The identification keys of Urals Myriapoda // Vestnik Permskogo universiteta. T.10. No.36. P.66-72 [in Russian].

Farzalieva G.Sh., Esyunin S.L. 2008. [A review of the centipede (Lithobiomorpha, Henicopidae, Lithobiidae) fauna of the Urals and Cisural Area] // Zoologicheskii Zhurnal. T.87. No.8. P.923947 [in Russian, with English summary].

Folkmanova B. 1956. [On new forms of the order Geophilomorpha from the southern regions of the USSR. To the knowledge of centipedes of the USSR] // Zoologicheskii Zhurnal. T.35. No.11. P.1633-1646 [in Russian, with English summary].

Folkmanova B. 1958. [On new forms of Chilopoda from the USSR] // Zoologicheskii Zhurnal. T.37. No.2. P.183-192 [in Russian, with German summary].

Folkmanova B., Dobroruka L.J. 1960. [To the knowledge of centipedes (Chilopoda) of the USSR] // Zoologicheskii Zhurnal. T.39. No.12. P.1811-1818 [in Russian, with German summary].

Ghilarov M.S., Folkmanova B. 1957. [Centipedes (Chilopoda) of the steppe zone of the southeastern European part of the USSR as indicators of soil conditions in forest plantations] // Izvestiya Akademii nauk SSSR, Seriya Biologicheskaya. T.2. P.211-219 [in Russian].

Kessler K.F. 1876. [On Russian Scolopendridae and Geophilidae] // Trudy Russkago Entomologicheskago obshchestva. St. Petersburg. T.8. P.28-45 [in Russian]

Kunah O.N. 2013. Chilopoda of the steppe Dnieper area // Biological Bulletin of Bogdan Chmelnitskiy Melitopol State Pedagogical University. T.1. No.7. P.65-81 [in Russian, with English summary].

Lignau N.G. 1903. Die Myriopoden der Pontus-Küsten von Caucasus // Zapiski Novorossiyskago Obshchestva estestvoispytateley. T.25. P.82-125 [in Russian and German].

Minoranskiy V.A. 1975. [To the fauna of centipedes of agrocenoses of the Rostov-on-Don Region] // Problemy pochvennoy zoologii. Materialy V Vsesoyuznogo soveshchaniya. Vilnius. P.229-231 [in Russian].

Minoranskiy V.A. 1977. Some data on Chilopoda (Myriapoda) from the Rostov Region // Vestnik zoologii. No.4. P.38-43 [in Russian, with English summary].
Minoranskiy V.A. 2004. Scolopendra cingulata // Krasnaya kniga Rostovskoy oblasti. T.I. P.45-46.

Muralewicz W.S. 1907. Zur Myriapodenfauna des Kaukasus. // Zoologischer Anzeiger. Bd.31. H.11-12. S.329-351.

Pereira L.A., Hoffman R.L. 1993. The American species of Escaryus, a genus of Holarctic centipeds (Geophilomorpha: Schendylidae) // Jeffersoniana. Vol.3. P.1-72.

Reip H.S., Voigtländer K. 2009. Diplopoda and Chilopoda of Thuringia // Soil Organisms. Vol.81. No.3. P.635-645.

Ruchin A.B. 2014. [To the myriapod (Myriapoda) fauna of Mordovia] // Molodoy uchenyi. T.2. P.387-390 [in Russian].

Simaiakis S.M., Zapparoli M., Minelli A., Bonato L. 2013. The centipede fauna (Chilopoda) of the island of Cyprus, with one new lithobiomorph species // Zootaxa. Vol.3647. No.2. P.279-306.

Sseliwanoff A.V. 1878. [Materials towards the study of Russian myriapods (Myriapoda)] // Trudy Russkago Entomologicheskago obshchestva. St. Petersburg. T.11. P.1-24 [in Russian].

Sseliwanoff A.V. 1883. [Materials towards the study of Russian myriapods] // Trudy Russkago Entomologicheskago obshchestva. St. Petersburg. T.18. P.69-121 [in Russian].

Stoev P. 2002. A catalogue and key to the centipedes (Chilopoda) of Bulgaria. Pensoft. 103 pp.

Titova L.P. 1969. [Geophilidae of the USSR fauna and new data on the family Mecistocephalidae distribution] // Problemy pochvennoy zoologii. Materialy III Vsesoyuznogo soveshchaniya. Kazan. P.165-166 [in Russian].

Titova L.P. 1972. [New species of the genus Escaryus Cook et Collins (Schendylidae Chilopoda)] // Ghilarov M.S. (Ed.). Ekologiya pochvennykh bespozvonochnykh, Moscow, Nauka. P. 94-119 [in Russian].

Titova L.P. 1975. [The centipede genera Clinopodes and Pleurogeophilus in the USSR fauna (Chilopoda, Geophilomorpha, Geophilidae)] // Problemy pochvennoy zoologii. Materialy V Vsesoyuznogo soveshchaniya. Vilnius. P. 308-309 [in Russian].

Volkova J.S. 2014a. [The centipede fauna of the Ulyanovsk Region] // Priroda Simbirskogo Povolzh'ya. Vyp.15. P.95-103 [in Russian].

Volkova J.S. 2014b. [Preliminary review of the myriapod (Myriapoda) fauna of the Saratov Region] // Entomological and parasitological investigations in the Volga region. Vyp.11. P.149-151 [in Russian].

Volkova J.S. 2015. [To the knowledge of myriapods (Myriapoda) of the Penza Region] // Priroda Simbirskogo Povolzh'ya. Vyp.16. P.72-75 [in Russian].

Zalesskaja N.T. 1973. [The lithobiids (Chilopoda, Lithobiidae) of the Caucasus, their distribution and connection to the soil] // Ghilarov M.S. (ed.). Ekologija pochvennykh bespozvonochnykh. Moscow: Nauka. P.120-130 [in Russian].

Zalesskaja N.T. 1978. [Identification book of the lithobiomorph centipedes of the USSR (Chilopoda, Lithobiomorpha)]. Moscow, Nauka. 212 pp. [in Russian].

Zalesskaja N. T., Golovatch S.I. 1996. Some patterns in the distribution and origin of the lithobiomorph centipede fauna of the Russian Plain (Chilopoda: Lithobiomorpha) // Geoffroy J.-J,, Mauriès J.-P., Nguyen Duy-Jacquemin M. (eds.). Acta Myriapodologica. Mémoires du Muséum national d'Histoire naturelle. N.S. T.169. P.265-268.

Zalesskaja N.T., Titova L.P., Golovatch S.I. 1982. [The myriapod fauna (Myriapoda) of the Moscow Area] // Ghilarov M.S. (ed.). Pochvennye bespozvonochnye Moskovskoy oblasti. Moscow: Nauka. P.179-200 [in Russian].

Zalesskaja N.T., Schileyko Ark. A. 1991. [Chilopods scolopendromorphs (Chilopoda, Scolopendromorpha)]. Moscow: Nauka. 102 p. [in Russian].

Zalesskaja N.T., Schileyko Ark. A. 1992. The distribution of Scolopendromorpha in the USSR (Chilopoda) // Meyer E., Thaler K., Schedl W. (eds.). Advances in Myriapodology. Berichte des naturwissenschaftlich-medizinischen Vereins in Innsbruck. Suppl.10. P.367-372.

Zapparoli M. 1999. The present knowledge of the centipede fauna of Anatolia (Chilopoda) // Biogeographia. Lavori delle Società Italiana di Biogeografia. T.20. P.105-177.

Zuev R.V. 2016. Centipedes (Chilopoda) from the Stavropol Territory, northern Caucasus, Russia // Arthropoda Selecta. Vol.25. No.1. P.23-38. 